\title{
Effect of hydro-priming and pre-germinating rice seed on the yield and terminal moisture stress mitigation of rain-fed lowland rice
}

\author{
Tilahun-Tadesse F. ${ }^{1, *}$, Nigussie-Dechassa . $^{2}$, Wondimu Bayu ${ }^{3}$, Setegn Gebeyehu ${ }^{4}$ \\ ${ }^{1}$ Amhra Region Agricultural Research Institute, P.O.Box 08, Bahir Dar, Ethiopia \\ ${ }^{2}$ Haramaya University, Department of Plant Sciences, P.O.Box 138, Dire Dawa, Ethiopia \\ ${ }^{3}$ ICARDA, Ethiopia \\ ${ }^{4}$ Ethiopian Institute of Agricultural Research, Ethiopia
}

\section{Email address:}

tilahuntade@yahoo.com (Tilahun-Tadesse F.), nigussiedachassa@gmail.com (Nigussie-Dechassa R.), wondimubayu@yahoo.com(W. Bayu), setegng@yahoo.co.uk (S. Gebeyehu)

\section{To cite this article}

Tilahun-Tadesse F., Nigussie-Dechassa R., Wondimu Bayu, Setegn Gebeyehu. Effect of Hydro-Priming and Pre-Germinating Rice Seed on the Yield and Terminal Moisture Stress Mitigation of Rain-Fed Lowland Rice, Agriculture, Forestry and Fisheries. Vol. 2, No. 2, 2013, pp. 89-97. doi: 10.11648/j.aff.20130202.15

\begin{abstract}
Terminal moisture stress is one of the major problems constraining rain-fed lowland rice production in northwestern Ethiopia. A field experiment was conducted in 2010 and 2011 at Fogera plains to study the effect of hydro-priming and pre-germinating rice seed on the yield and response of the crop to terminal moisture stress. A factorial combination of eight seed treatments and three sowing times were studied in RCB design with three replications. Data on crop phenology, shoot biomass, leaf area, number of productive and unproductive tillers, filled and unfilled spikeletes, plant height, biomass yield, thousand seed weight, and grain yield were collected and analyzed. The results revealed that planting pre-germinated seeds as well as seeds soaked and dried for $24 \mathrm{hrs}$ at the local (farmers') sowing time resulted in significantly earlier seedling emergence, heading, and maturity. Higher numbers of productive tillers, filled spikeletes, leaf area index, crop growth rate, net assimilation rate, grain yield, biomass yield, and harvest index were recorded in response to planting pregerminated seeds followed by seeds soaked and dried for $24 \mathrm{hrs}$ at farmers' sowing time. The present study concluded that planting pre-germinated seeds or hydro-primed seeds soaked and dried for $24 \mathrm{hrs}$ could be practiced as the first and second best alternatives for rice production on Fogera plains in northwestern Ethiopia.
\end{abstract}

Keywords: Hydro-Priming, Lowland Rice, Moisture Stress, Pre-Germination, Rain-Fed

\section{Introduction}

Rice (Oryza sativa L.) is one of the most important food cereal crops in the world. The crop is a staple food for nearly half of the world's population. It is mostly grown in lowlands under full irrigation or rain-fed conditions. Rain-fed lowland rice occupies approximately $35 \%$ of global rice area [1]. Most rain-fed lowlands are frequently constrained by drought [2]. Moisture stress during the latter rice growth phases appears to affect pollination, fertilization and grain filling. To curb the challenges of terminal moisture stress, establishment of irrigation facilities and rainwater harvesting as well as developing drought-tolerant and short duration crop varieties are useful remedial measures [2]. According to Ceesay [3] and Hassanein et al. [4], seed priming and pre-germination are agronomic techniques that could enable the crop to give higher yields under terminal moisture stress conditions. Pre-germinated and primed seeds generally induce the establishment of more rapid and vigorous rice seedling and earlier flowering and maturity of the crop than dry seeds [5]. Early flowering and maturity allow the crop to escape terminal drought [5].

Pre-germination is a pre-sowing process whereby rice seeds are soaked in water for some time and taken out to remain incubated in a moist shaded place until the seed starts germinating [6]. The pre-germinated seeds sprout more quickly compared to dry seed thereby reducing the time of exposure to different environmental stresses that may affect seedling development. In Ethiopia, Tilahun et al. [7] recommended 48-hour soaking and one day incubation for rice. Pre-germinated seeds need to be planted imme- 
diately in a moist soil. In a tropical climate, moisture stress at planting could result in poor [6]. Under such conditions, seed priming might work better [6]. In seed priming, seeds are partially hydrated to a point where germination-related metabolic processes begin but just prior to germination where radicle emergence does not occur [8]. After priming, seeds are air-dried back near to the original weight [8]. According to Farooq et al. [8], seeds can be primed using different media such as tap water (hydro-priming), low water potential solutions (osmo-priming) such as polyethylene glycol or salt solutions $\left(\mathrm{KNO}_{3}, \mathrm{KCl}, \mathrm{MgSO}_{4}, \mathrm{CaCl}_{2}\right.$ and $\mathrm{NaCl}$ ), solid matrix (matri-priming), and plant growth regulators (hormonal priming). Harris et al. [9] indicated that hydro-priming is the best option for smallholder farmers in developing countries since it is a low cost and low risk intervention.

Seeds that underwent priming increased their total sugar and $\alpha$-amylase activity and exhibited earlier initiation of protein, RNA, and DNA synthetic activity. Consequently, when the seed is set out for germination, cellular events are much advanced [10]. Primed seeds behave as dry seeds if sowing is delayed or seedbed conditions are suboptimal [10]. Hydro-primed rice seeds stored for one month under ambient storage conditions were found to retain the ability for increased germination rates. Primed seeds would not germinate until they have taken up additional water from a moist seedbed whereas pre-germinated seeds would continue germinating regardless of external soil moisture conditions $[9,10]$. Success of seed priming is influenced by the duration of priming until the optimum hours. For every crop species, there is a 'safe limit', the maximum length of time, if exceeded, could lead to seed damage [10]. Thakur et al. [6] recommended 15 to 18 hours hydro-priming duration for rice. On the other hand, Harris et al. [9] recommended a 24-hr safe limit for rice with only minor varietal differences. Therefore, this study was conducted with the objective of assessing the effects of seed priming and seed pre-germination on mitigating terminal moisture stress on the growth and yield of rice crop.

\section{Materials and Methods}

\subsection{The Study Site}

The study was conducted in Fogera plain located at $13^{0}$ 19' latitude, $37^{\circ} 03^{\prime}$ longitude and at the altitude of 1815 meters above sea level in northwestern Ethiopia in the 2010 and 2011 cropping seasons. Eleven-year (2001-2011) meteorological data of the area indicates that in the main cropping season (June-October) the area had mean annual minimum and maximum temperatures of $13.5^{\circ} \mathrm{C}$ and $26.1^{\circ} \mathrm{C}$, respectively. The area received an annual rainfall of 1205 $\mathrm{mm}$ which occurs from June to October. The soil is Vertisol with a clay content of $71.25 \%$. It is slightly acidic ( $\mathrm{pH} 5.90)$ and the $20 \mathrm{~cm}$ soil horizon contains $0.22 \%$ total $\mathrm{N}, 12.64$ ppm available $\mathrm{P}$ (Olsen), $0.93 \mathrm{cmol}(+) \mathrm{kg}^{-1}$ exchangeable $\mathrm{K}$, $3 \%$ organic carbon and $52.9 \mathrm{cmol}(+) \mathrm{kg}^{-1}$ CEC. According to Bernard [11], the total $\mathrm{N}$ and available $\mathrm{P}$ contents of the soil are medium while the organic matter content is low. According to Roy et al [12], the exchangeable potassium content and CEC are high.

\subsection{Experimental Design and Procedures}

Treatments comprised a factorial combination of eight seed treatments and three sowing dates. The experimental design was RCB in three replications. The eight seed treatments were dry seeds (ST1, control), pre-germinated seeds after a 48-hour seed soaking and a 24-hour incubation (ST2), seeds soaked for 12 hours and dried for 24 hours (ST3), seeds soaked for 18 hours and dried for 24 hours (ST4), seeds soaked for 24 hours and dried for 24 hours (ST5), seeds soaked for 12 hours, dried for 24 hours, and then resoaked for 12 hours and dried for 24 hours (ST6), seeds soaked for 18 hours and dried for 24 hours and then resoaked for 18 hours and dried for 24 hours (ST7), and seeds soaked for 24 hours and dried for 24 hours and then re-soaked for $24 \mathrm{hrs}$ and dried for one day (ST8). Seed soaking was done in tap water. The three sowing dates were: sowing two weeks before farmers' sowing time (SD1), sowing one week before farmers' sowing time (SD2), and sowing during farmers' sowing time (SD3). The farmers sowing dates were 14-24 June in 2010 and 20-30 June in 2011. Seeds of a rice variety, called X-Jigna, were broadcasted at the seed rate of $140 \mathrm{~kg} \mathrm{ha}^{-1}$. Fertilizers at the rates of $69-23 \mathrm{~N}_{-} \mathrm{P}_{2} \mathrm{O}_{5} \mathrm{~kg} \mathrm{ha}{ }^{-1}$ were applied. Weeds were removed by hand weeding three times (at early tillering, maximum tillering, and booting stages). No insecticide or fungicide was applied since no serious insect or disease incidences occurred. Harvesting was done manually using hand sickles. Gross and net plot sizes were $3 \mathrm{~m} \mathrm{x} 4 \mathrm{~m}$ and 2 $\mathrm{m} \times 3 \mathrm{~m}$, respectively.

Data on the number of days to emergence as well as on the number of heading and maturity were collected. Leaf area at heading was measured using the mathematical equation developed by Yoshida [13]:

$$
\text { Leaf area }\left(\mathrm{cm}^{2}\right)=\mathrm{L} \times \mathrm{W} \times K
$$

Where, $\mathrm{L}$ is the leaflength, $\mathrm{W}$ is the maximum width of the leaf and $\mathrm{K}$ is a correction factor of 0.75 .

Leaf area index (LAI) was also calculated using the formula of Yoshida [13]:

$$
\mathrm{LAI}=
$$

Sum of the leaf area of all leaves

Ground area of field where the leaves have been collected

Crop Growth Rate (CGR) and Net Assimilation Rate (NAR) for the duration from planting to heading were computed using the equations developed by Hunt [14] as cited by Ahmad et al. [15]:

$$
\mathrm{CGR}=\frac{1}{A} X\left(\frac{\Delta D M}{\Delta T}\right) \text { and } \mathrm{NAR}=\frac{1}{L A} X\left(\frac{\Delta D M}{\Delta T}\right)
$$

Where, $\mathrm{A}$ is area of land, $\Delta D M$ is change in Dry matter, 
$\Delta T$ is time variations in day, LA is total leaf area per unit area of land. CGR is expressed as $\mathrm{g}$ dry matter $\mathrm{m}-2$ land area day-1and NAR is expressed as $g$ dry matter $\mathrm{m}-2$ leaf area day-1 [15].

Data on the number of productive and unproductive tillers per $\mathrm{m}-2$, filled and unfilled spikeletes plant-1, plant height, thousand seed weight, grain yield, and aboveground biomass yield were collected. Data recorded on plant basis were measured from 15 randomly selected plants within the net plot area in each plot. Grain yield was adjusted to $14 \%$ moisture content. Harvest index (HI) was calculated as the ratio of grain yield to aboveground biomass yield. The data were subjected to analysis of variance using SAS software [16]. Homogeneity of error variances was tested using $\mathrm{F}$ test as described by [17] and the F-test was not significant.
Thus, combined analysis of the two years data was performed. Differences among treatment means were delineated using the least significant difference $(\mathrm{P}<0.05)$ test.

\section{Results}

The rainfall data indicated that in the second year of the experiment (2011) the crop suffered from terminal moisture stress since rain did not fall in September and was extremely low in October (Table 1). The total amount of rainfall received during the growing season of 2011 was much lower than the expected amount compared to the amount rainfall received during the remaining period of the year.

Table 1. Ten-year (2002-2011) rainfall data ( $\mathrm{mm}$ ) for the growing season at Fogera.

\begin{tabular}{lllllll}
\hline Year & June & July & August & September & October & Total \\
\hline 2002 & 309.1 & 327.3 & 335.4 & 115.2 & 13.4 & 1100.4 \\
2003 & 245.2 & 301.4 & 404.4 & 292.9 & 9.5 & 1253.4 \\
2004 & 163.1 & 362.1 & 402.6 & 120.5 & 55.6 & 1103.9 \\
2005 & 262.1 & 261.8 & 461.6 & 271.3 & 44.6 & 1301.4 \\
2006 & 118.9 & 388.7 & 503.0 & 180.3 & 96.6 & 1287.5 \\
2007 & 167.5 & 232.3 & 425.0 & 346.7 & 7.0 & 1178.5 \\
2008 & 247.2 & 519.1 & 486.6 & 292.2 & 7.5 & 1552.6 \\
2009 & 121.8 & 400.5 & 356.5 & 159.4 & 64.0 & 1102.2 \\
2010 & 119.4 & 526.5 & 426.6 & 21.5 & 1.2 & 1095.2 \\
2011 & 71.7 & 394.1 & 341.9 & 0.0 & 9.5 & 817.2 \\
\multicolumn{5}{l}{ Source: National Meteorology Agency of Ethiopia, Bahir Dar Branch } \\
\hline
\end{tabular}

Days to emergence, days to heading, and days to maturity were significantly affected by the seed treatment, sowing date as well as by their interactions (Table 2). Seedlings emerged significantly earlier when pregerminated seeds were planted on farmers' sowing date. This was closely followed by the emergence of seedlings from seeds soaked for $24 \mathrm{hrs}$ and dried for $24 \mathrm{hrs}$ that were sown in accordance with farmers' sowing time (Table 3). Emergence dates were reduced by about $51 \%$ and $35 \%$ with these treatments, respectively. Days to heading and maturity were also reached significantly earlier when pre-germinated seeds were planted according to farmers' sowing date. This was also closely followed by seedlings that emerged from seeds that were soaked for $24 \mathrm{hrs}$ and dried for the same duration of time with the same sowing date. Thus, the maturity date of plants emerging from the pre-germinated seeds was earlier by about 12.8-14.1 days compared to the plants that grew from untreated (control plot) seeds (Tables 3 and 4).

Table 2. Mean square values from analysis of variance (ANOVA) for the effect of seed treatment and sowing date on the growth parameters of rice at Fogera plain in 2010 and 2011.

\begin{tabular}{|c|c|c|c|c|}
\hline \multirow{2}{*}{ Growth parameters } & \multicolumn{4}{|l|}{ Mean squares } \\
\hline & Seed treatment $(\mathrm{ST})$ & Sowing date (SD) & ST $x$ SD & Error Mean Square \\
\hline Days to emergence & $17.0^{*}$ & $384.0^{*}$ & $5.2 *$ & 1.906 \\
\hline Days to heading & $30.8^{*}$ & $1784.5^{*}$ & $8.1 *$ & 1.976 \\
\hline Days to maturity & $57.4^{*}$ & $1259.6^{*}$ & $12.6^{*}$ & 2.728 \\
\hline Leaf area index (LAI) at heading & $12.3^{*}$ & $15.2 *$ & $13.4 *$ & 1.249 \\
\hline Crop growth rate (CGR) & $954.5^{*}$ & $325.7^{*}$ & $745.1^{*}$ & 67.05 \\
\hline Net assimilation rate (NAR) & $193.0^{*}$ & $196.1^{*}$ & $182.2^{*}$ & 10.341 \\
\hline Number of productive tillers $\mathrm{m}^{-2}$ & $81680.6^{*}$ & $15830.4 *$ & $22107.6^{*}$ & 3705.6 \\
\hline Number of unproductive tillers per $\mathrm{m}^{-2}$ & $286.7^{*}$ & $325.0^{*}$ & $109.7 *$ & 16.96 \\
\hline Number of filled spikeletes plant ${ }^{-1}$ & $6184.3 *$ & $43350.1^{*}$ & $1437.0^{*}$ & 27.471 \\
\hline Number of unfilled spikeletes plant ${ }^{-1}$ & $433.9^{*}$ & $3582.9^{*}$ & $81.3 *$ & 11.574 \\
\hline
\end{tabular}


Tilahun-Tadesse F. et al.: Effect of hydro-priming and pre-germinating rice seed on the yield and terminal moisture stress mitigation of rain-fed lowland rice

Table 3. Effect of seed treatment and sowing time on days to emergence and heading of rice at Fogera in 2010 and 2011.

\begin{tabular}{|c|c|c|c|c|c|c|}
\hline \multirow{3}{*}{ Seed treatment } & \multicolumn{3}{|c|}{ Days to emergence } & \multicolumn{3}{|c|}{ Days to heading } \\
\hline & \multicolumn{3}{|c|}{ Sowing time relative to farmers' time } & \multicolumn{3}{|c|}{ Sowing time relative to farmers' time } \\
\hline & 2 weeks before & 1 week before & $\begin{array}{l}\text { Farmers' sowing } \\
\text { time }\end{array}$ & $\begin{array}{l}2 \text { weeks } \\
\text { before }\end{array}$ & 1 week before & $\begin{array}{l}\text { Farmers' sowing } \\
\text { time }\end{array}$ \\
\hline Dry seed (control) & $19.3^{\mathrm{A}}$ & $14.0^{\mathrm{C}}$ & $12.3^{\mathrm{CDE}}$ & $112.7^{\mathrm{A}}$ & $98.0^{\mathrm{FG}}$ & $99.3^{\mathrm{FG}}$ \\
\hline Pregerminated & $18.0^{\mathrm{AB}}$ & $10.7^{\mathrm{FG}}$ & $6.0^{\mathrm{H}}$ & $112.3^{\mathrm{AB}}$ & $92.3^{\mathrm{JK}}$ & $90.7^{\mathrm{K}}$ \\
\hline 12 hrs soaking $+24 \mathrm{hr}$ drying & $18.0^{\mathrm{AB}}$ & $13.7^{\mathrm{C}}$ & $10.7^{\mathrm{EF}}$ & $110.3^{\mathrm{BCD}}$ & $100.0^{\mathrm{F}}$ & $99.7^{\mathrm{F}}$ \\
\hline 18 hrs soaking $+24 \mathrm{hr}$ drying & $17.7^{\mathrm{AB}}$ & $13.0^{\mathrm{CD}}$ & $10.3^{\mathrm{EF}}$ & $110.7^{\mathrm{ABC}}$ & $97.3^{\mathrm{GH}}$ & $95.0^{\mathrm{HI}}$ \\
\hline 24 hrs soaking $+24 \mathrm{hr}$ drying & $17.3^{\mathrm{AB}}$ & $13.0^{\mathrm{CD}}$ & $8.0^{\mathrm{GH}}$ & $109.7^{\mathrm{CDE}}$ & $97.7^{\mathrm{FG}}$ & $92.1^{\mathrm{JK}}$ \\
\hline $\begin{array}{l}12 \mathrm{hrs} \text { soaking }+24 \mathrm{hr} \text { drying }+ \\
12 \mathrm{hrs} \text { resoaking }+24 \mathrm{hr} \text { drying }\end{array}$ & $17.7^{\mathrm{AB}}$ & $12.3^{\mathrm{CDE}}$ & $9.1^{\mathrm{FG}}$ & $109.0^{\mathrm{CDE}}$ & $95.0^{\mathrm{HI}}$ & $94.0^{\mathrm{IJ}}$ \\
\hline $\begin{array}{l}18 \mathrm{hrs} \text { soaking }+24 \mathrm{hr} \text { drying }+ \\
18 \mathrm{hrs} \text { resoaking }+24 \mathrm{hr} \text { drying }\end{array}$ & $16.3^{\mathrm{B}}$ & $12.3^{\mathrm{CDE}}$ & $9.0^{\mathrm{FG}}$ & $108.0^{\mathrm{DE}}$ & $95.3^{\mathrm{HI}}$ & $93.5^{\mathrm{IJ}}$ \\
\hline $\begin{array}{l}24 \mathrm{hrs} \text { soaking }+24 \mathrm{hr} \text { drying }+ \\
24 \mathrm{hrs} \text { resoaking }+24 \mathrm{hr} \text { drying }\end{array}$ & $18.1^{\mathrm{AB}}$ & $11.0^{\mathrm{DEF}}$ & $10.0^{\mathrm{FG}}$ & $107.7^{\mathrm{E}}$ & $94.3^{\mathrm{IJ}}$ & $93.7^{\mathrm{IJ}}$ \\
\hline CV (\%) & 10.59 & & & 11.32 & & \\
\hline
\end{tabular}

Table 4. Effect of seed treatment and sowing time on days to maturity and LAI of rice at Fogera in 2010 and 2011.

\begin{tabular}{|c|c|c|c|c|c|c|}
\hline \multirow{3}{*}{ Seed treatment } & \multicolumn{3}{|l|}{ Days to maturity } & \multicolumn{3}{|l|}{ LAI } \\
\hline & \multicolumn{3}{|c|}{ Sowing time relative to farmers' time } & \multicolumn{3}{|c|}{ Sowing time relative to farmers' time } \\
\hline & 2 weeks before & 1 week before & $\begin{array}{l}\text { Farmers' sowing } \\
\text { time }\end{array}$ & $\begin{array}{l}2 \text { weeks } \\
\text { before }\end{array}$ & 1 week before & $\begin{array}{l}\text { Farmers' sowing } \\
\text { time }\end{array}$ \\
\hline Dry seed (control) & $136.3^{\mathrm{A}}$ & $134.3^{\mathrm{AB}}$ & $132.8^{\mathrm{BC}}$ & $2.7^{\mathrm{G}}$ & $4.7^{\mathrm{A}-\mathrm{E}}$ & $5.2^{\mathrm{A}-\mathrm{E}}$ \\
\hline Pregerminated & $134.2^{\mathrm{AB}}$ & $124.0^{\mathrm{IJ}}$ & $118.7^{\mathrm{K}}$ & $2.9^{\mathrm{FG}}$ & $5.0^{\mathrm{A}-\mathrm{E}}$ & $6.3^{\mathrm{A}}$ \\
\hline 12 hrs soaking +24 hr drying & $132.7^{\mathrm{BCD}}$ & $128.7^{\mathrm{EF}}$ & $125.3^{\mathrm{H}-\mathrm{J}}$ & $4.5^{\mathrm{B}-\mathrm{F}}$ & $5.0^{\mathrm{A}-\mathrm{E}}$ & $5.4^{\mathrm{A}-\mathrm{D}}$ \\
\hline $18 \mathrm{hrs}$ soaking $+24 \mathrm{hr}$ drying & $133.3^{\mathrm{BC}}$ & $130.0^{\mathrm{DE}}$ & $124.3^{\mathrm{HIJ}}$ & $3.7^{\mathrm{EFG}}$ & $4.9^{\mathrm{A}-\mathrm{E}}$ & $6.2^{\mathrm{A}}$ \\
\hline 24 hrs soaking +24 hr drying & $134.7^{\mathrm{AB}}$ & $127.0^{\mathrm{FGH}}$ & $120.0^{\mathrm{JK}}$ & $4.6^{\mathrm{B}-\mathrm{F}}$ & $6.0^{\mathrm{ABC}}$ & $6.1^{\mathrm{AB}}$ \\
\hline $\begin{array}{l}12 \mathrm{hrs} \text { soaking }+24 \mathrm{hr} \text { drying }+ \\
12 \mathrm{hrs} \text { resoaking }+24 \mathrm{hr} \text { drying }\end{array}$ & $135.3^{\mathrm{AB}}$ & $128.0^{\mathrm{EFG}}$ & $123.3^{\mathrm{J}}$ & $4.2^{\mathrm{D}-\mathrm{G}}$ & $4.7^{\mathrm{A}-\mathrm{E}}$ & $6.0^{\mathrm{ABC}}$ \\
\hline $\begin{array}{l}18 \mathrm{hrs} \text { soaking }+24 \mathrm{hr} \text { drying }+ \\
18 \mathrm{hrs} \text { resoaking }+24 \mathrm{hr} \text { drying }\end{array}$ & $133.7^{\mathrm{AB}}$ & $126.7^{\mathrm{F}-\mathrm{I}}$ & $124.7^{\mathrm{HIJ}}$ & $4.4^{\mathrm{C}-\mathrm{F}}$ & $5.3^{\mathrm{A}-\mathrm{E}}$ & $5.7^{\mathrm{A}-\mathrm{D}}$ \\
\hline $\begin{array}{l}24 \mathrm{hrs} \text { soaking }+24 \mathrm{hr} \text { drying }+ \\
24 \mathrm{hrs} \text { resoaking }+24 \mathrm{hr} \text { drying }\end{array}$ & $130.7^{\mathrm{CDE}}$ & $125.0^{\mathrm{HIJ}}$ & $125.0^{\mathrm{HIJ}}$ & $4.5^{\mathrm{B}-\mathrm{F}}$ & $5.6^{\mathrm{A}-\mathrm{D}}$ & $5.8^{\mathrm{A}-\mathrm{D}}$ \\
\hline CV $(\%)$ & 11.14 & & & 22.31 & & \\
\hline
\end{tabular}

LAI significantly responded to the seed treatment, sowing time and their interaction effects (Table 2). All of the treatments planted two weeks before the farmers sowing date exhibited lower LAI at heading (Table 4). Though all 
the treatments planted a week before and at the farmers' sowing time resulted in statistically equivalent LAI at heading, numerically highest LAI of 6.3 was observed with sowing pre-germinated seeds at farmers' planting time (Table 4). CGR responded to the seed treatment, sowing time and interaction effects (Table 2). Except the dry seed, all the seed treatments planted both a week before and at the farmers sowing time resulted in statistically equivalent and higher CGR values (Table 5). Dry seeds across the three sowing times and all the seed treatments planted two weeks before the farmers sowing time resulted in statistically lower CGR values (Table 5). NAR had significant response to the seed treatment, sowing time and interaction effects (Table 2). Statistically highest NAR values were recorded for pre-germinated seeds, and for hydro-primed seeds after a 24-hr seed soaking and drying for 24 hours both planted at farmers' sowing time (Table 5). The lowest NAR values were recorded when dry seeds and pre-germinated seeds were planted two weeks before farmers' sowing time.

Table 5. Effect of seed treatment and sowing time on CGR and NAR of rice at Fogera in 2010 and 2011 main cropping season.

\begin{tabular}{|c|c|c|c|c|c|c|}
\hline \multirow{3}{*}{ Seed treatment } & \multicolumn{3}{|c|}{ CGR (g dry matter $m^{-2}$ land area day ${ }^{-1}$ ) } & \multicolumn{3}{|c|}{ NAR (g dry matter $m^{-2}$ leaf area day ${ }^{-1}$ ) } \\
\hline & \multicolumn{3}{|c|}{ Sowing time relative to farmers' time } & \multicolumn{3}{|c|}{ Sowing time relative to farmers' time } \\
\hline & 2 weeks before & 1 week before & $\begin{array}{l}\text { Farmers' sowing } \\
\text { time }\end{array}$ & $\begin{array}{l}2 \text { weeks } \\
\text { before }\end{array}$ & 1 week before & $\begin{array}{l}\text { Farmers' sowing } \\
\text { time }\end{array}$ \\
\hline Dry seed (control) & $6.2^{\mathrm{E}}$ & $7.8^{\mathrm{E}}$ & $11.2^{\mathrm{DE}}$ & $2.5^{\mathrm{G}}$ & $4.6^{\mathrm{E}-\mathrm{F}}$ & $4.6^{\mathrm{E}-\mathrm{F}}$ \\
\hline Pregerminated & $4.6^{\mathrm{E}}$ & $31.8^{\mathrm{ABC}}$ & $37.5^{\mathrm{A}}$ & $2.0^{\mathrm{G}}$ & $5.0^{\mathrm{D}-\mathrm{E}}$ & $6.1^{\mathrm{A}}$ \\
\hline 12 hrs soaking $+24 \mathrm{hr}$ drying & $14.8^{\mathrm{CDE}}$ & $21.0^{\mathrm{A}-\mathrm{E}}$ & $20.7^{\mathrm{A}-\mathrm{E}}$ & $4.6^{\mathrm{EF}}$ & $4.7^{\mathrm{EF}}$ & $4.8^{\mathrm{DEF}}$ \\
\hline 18 hrs soaking $+24 \mathrm{hr}$ drying & $7.4^{\mathrm{E}}$ & $21.4^{\mathrm{A}-\mathrm{E}}$ & $19.9^{\mathrm{A}-\mathrm{E}}$ & $4.2^{\mathrm{F}}$ & $4.9^{\mathrm{DE}}$ & $5.2^{\mathrm{CDE}}$ \\
\hline 24 hrs soaking $+24 \mathrm{hr}$ drying & $14.5^{\mathrm{CDE}}$ & $26.2^{\mathrm{A}-\mathrm{D}}$ & $34.0^{\mathrm{AB}}$ & $4.9^{\mathrm{DE}}$ & $4.8^{\mathrm{DEF}}$ & $5.9^{\mathrm{AB}}$ \\
\hline $\begin{array}{l}12 \mathrm{hrs} \text { soaking }+24 \mathrm{hr} \text { drying }+ \\
12 \mathrm{hrs} \text { resoaking }+24 \mathrm{hr} \text { drying }\end{array}$ & $18.1^{\mathrm{B}-\mathrm{E}}$ & $20.6^{\mathrm{A}-\mathrm{E}}$ & $22.0^{\mathrm{A}-\mathrm{E}}$ & $5.0^{\mathrm{DE}}$ & $5.0^{\mathrm{DE}}$ & $5.4^{\mathrm{BCD}}$ \\
\hline $\begin{array}{l}18 \mathrm{hrs} \text { soaking }+24 \mathrm{hr} \text { drying }+ \\
18 \mathrm{hrs} \text { resoaking }+24 \mathrm{hr} \text { drying }\end{array}$ & $15.0^{\mathrm{CDE}}$ & $29.6^{\mathrm{ABC}}$ & $31.8^{\mathrm{ABC}}$ & $4.8^{\mathrm{DEF}}$ & $5.1^{\mathrm{DE}}$ & $5.0^{\mathrm{DE}}$ \\
\hline $\begin{array}{l}24 \mathrm{hrs} \text { soaking }+24 \mathrm{hr} \text { drying }+ \\
24 \mathrm{hrs} \text { resoaking }+24 \mathrm{hr} \text { drying }\end{array}$ & $15.3^{\mathrm{CDE}}$ & $31.0^{\mathrm{ABC}}$ & $32.6^{\mathrm{ABC}}$ & $4.7^{\mathrm{EF}}$ & $4.9^{\mathrm{DE}}$ & $5.1^{\mathrm{CDE}}$ \\
\hline CV (\%) & 14.98 & & & 21.49 & & \\
\hline
\end{tabular}

The number of rice productive (panicle bearing) and unproductive (without panicles) tillers $\mathrm{m}-2$, and number of filled and unfilled spikelets plant-1 had significant response to the main treatment effects and interaction effects (Table 2). The highest number of productive tillers and filled spikelets and the lowest numbers of unproductive tillers and unfilled spikelets were recorded for pregerminated seeds and seeds soaked for $24 \mathrm{hrs}$ and dried for the same duration both planted at farmers' sowing time (Tables 6 and 7). The plant height and thousand seed weight were not significantly affected by seed and sowing time treatments as well as by their interactions (Table 8). Aboveground dry biomass significantly responded to seed treatment and sowing time treatments as well as to their interactions (Table 8). The highest aboveground dry biomass was realized for most of the seed treatments planted at the farmers' sowing time, except for dry seeds and hydro-primed seeds by soaked for 12 hours and dried for 24 hours (Table 9). Grain yield and harvest index were significantly affected by the interaction effects of seed treatment and sowing time (Table 8 ). The highest grain yields were recorded when pregerminated seeds were planted at farmers' sowing time followed by planting seeds soaked for $24 \mathrm{hrs}$ and dried for $24 \mathrm{hrs}$ at farmers' planting time (Tables 9). Planting pre-germinated seeds at farmers' sowing time resulted in the yield advantage of $1.73 \mathrm{t}$ ha-1 over planting dry seeds at similar sowing time. Grain yields were the lowest for all seed treatments when planted two weeks before farmers' sowing time (Tables 9). Except the dry seed, all treated seeds planted both a week before and at the farmers' sowing time had statistically equivalent and higher harvest indices (HI) (Table 10). Dry seeds across the three sowing times and all seed treatments planted two weeks before the farmers' sowing time had significantly lower HI values (Table 10). 
Table 6. Effect of seed treatment and sowing time on number of productive and unproductive tillers (m-2) of rice at Fogera in 2010 and 2011 main cropping season.

\begin{tabular}{|c|c|c|c|c|c|c|}
\hline \multirow{3}{*}{ Seed treatment } & \multicolumn{3}{|c|}{ Number of productive tillers } & \multicolumn{3}{|c|}{ Number of unproductive tillers } \\
\hline & \multicolumn{3}{|c|}{ Sowing time relative to farmers' time } & \multicolumn{3}{|c|}{ Sowing time relative to farmers' time } \\
\hline & 2 weeks before & 1 week before & $\begin{array}{l}\text { Farmers' sowing } \\
\text { time }\end{array}$ & $\begin{array}{l}2 \text { weeks } \\
\text { before }\end{array}$ & 1 week before & $\begin{array}{l}\text { Farmers' sowing } \\
\text { time }\end{array}$ \\
\hline Dry seed (control) & $109^{\mathrm{GH}}$ & $119^{\mathrm{FGH}}$ & $133^{\mathrm{E}-\mathrm{H}}$ & $39.3^{\mathrm{A}}$ & $23.0^{\mathrm{D}}$ & $18.3^{\mathrm{D}-\mathrm{I}}$ \\
\hline Pregerminated & $98^{\mathrm{H}}$ & $209^{\mathrm{CF}}$ & $370^{\mathrm{A}}$ & $33.7^{\mathrm{B}}$ & $12.3^{\mathrm{KL}}$ & $7.0^{\mathrm{M}}$ \\
\hline $12 \mathrm{hrs}$ soaking $+24 \mathrm{hr}$ drying & $120^{\mathrm{FGH}}$ & $131^{\mathrm{E}-\mathrm{H}}$ & $137^{\mathrm{D}-\mathrm{H}}$ & $28.7^{\mathrm{C}}$ & $21.0^{\mathrm{DEE}}$ & $17.7^{\mathrm{E}-\mathrm{J}}$ \\
\hline 18 hrs soaking $+24 \mathrm{hr}$ drying & $108^{\mathrm{GH}}$ & $104^{\mathrm{GH}}$ & $185^{\mathrm{C}-\mathrm{H}}$ & $23.0^{\mathrm{D}}$ & $20.3^{\mathrm{DEF}}$ & $21.0^{\mathrm{DEF}}$ \\
\hline 24 hrs soaking $+24 \mathrm{hr}$ drying & $119^{\mathrm{FGH}}$ & $151^{\mathrm{D}-\mathrm{H}}$ & $313^{\mathrm{AB}}$ & $22.3^{\mathrm{DE}}$ & $14.0^{\mathrm{I}-\mathrm{L}}$ & $9.8^{\mathrm{LM}}$ \\
\hline $\begin{array}{l}12 \mathrm{hrs} \text { soaking }+24 \mathrm{hr} \text { drying }+ \\
12 \mathrm{hrs} \text { resoaking }+24 \mathrm{hr} \text { drying }\end{array}$ & $182^{\mathrm{C}-\mathrm{H}}$ & $198^{\mathrm{C}-\mathrm{G}}$ & $197^{\mathrm{C}-\mathrm{G}}$ & $19.5^{\mathrm{D}-\mathrm{G}}$ & $19.3^{\mathrm{D}-\mathrm{H}}$ & $14.7^{\mathrm{H}-\mathrm{K}}$ \\
\hline $\begin{array}{l}18 \mathrm{hrs} \text { soaking }+24 \mathrm{hr} \text { drying }+ \\
18 \mathrm{hrs} \text { resoaking }+24 \mathrm{hr} \text { drying }\end{array}$ & $208^{\mathrm{C}-\mathrm{F}}$ & $230^{\mathrm{BCD}}$ & $273^{\mathrm{BC}}$ & $17.8^{\mathrm{E}-\mathrm{J}}$ & $15.3^{\mathrm{G}-\mathrm{K}}$ & $13.3^{\mathrm{JKL}}$ \\
\hline $\begin{array}{l}24 \mathrm{hrs} \text { soaking }+24 \mathrm{hr} \text { drying }+ \\
24 \mathrm{hrs} \text { resoaking }+24 \mathrm{hr} \text { drying }\end{array}$ & $152^{\mathrm{D}-\mathrm{H}}$ & $226^{\mathrm{B}-\mathrm{E}}$ & $252^{\mathrm{BC}}$ & $17.7^{\mathrm{E}-\mathrm{J}}$ & $16.7^{\mathrm{F}-\mathrm{K}}$ & $12.2^{\mathrm{KL}}$ \\
\hline CV (\%) & 11.5 & & & 12.62 & & \\
\hline
\end{tabular}

Table 7. Effect of seed treatment and sowing time on number of filled and unfilled spikeletes plant-1 at Fogera in 2010 and 2011 cropping season.

\begin{tabular}{|c|c|c|c|c|c|c|}
\hline \multirow{3}{*}{ Seed treatment } & \multicolumn{3}{|c|}{ Number of filled spikeletes } & \multicolumn{3}{|c|}{ Number of unfilled spikeletes } \\
\hline & \multicolumn{3}{|c|}{ Sowing time relative to farmers' time } & \multicolumn{3}{|c|}{ Sowing time relative to farmers' time } \\
\hline & 2 weeks before & 1 week before & $\begin{array}{l}\text { Farmers' sowing } \\
\text { time }\end{array}$ & $\begin{array}{l}2 \text { weeks } \\
\text { before }\end{array}$ & 1 week before & $\begin{array}{l}\text { Farmers' sowing } \\
\text { time }\end{array}$ \\
\hline Dry seed (control) & $62^{\mathrm{MN}}$ & $119^{\mathrm{K}}$ & $126^{\mathrm{JK}}$ & $67^{\mathrm{A}}$ & $36^{\mathrm{EF}}$ & $34^{\mathrm{EFG}}$ \\
\hline Pregerminated & $54^{\mathrm{NO}}$ & $152^{\mathrm{EFG}}$ & $197^{\mathrm{A}}$ & $58^{\mathrm{B}}$ & $32^{\mathrm{E}-\mathrm{H}}$ & $18^{\mathrm{K}}$ \\
\hline 12 hrs soaking $+24 \mathrm{hr}$ drying & $62^{\mathrm{MN}}$ & $148^{\mathrm{GHI}}$ & $149^{\mathrm{FGH}}$ & $52^{\mathrm{C}}$ & $34^{\mathrm{EFG}}$ & $31^{\mathrm{FGH}}$ \\
\hline 18 hrs soaking $+24 \mathrm{hr}$ drying & $65^{\mathrm{LM}}$ & $143^{\mathrm{HI}}$ & $157^{\mathrm{EF}}$ & $51^{\mathrm{C}}$ & $30^{\mathrm{GH}}$ & $30^{\mathrm{GH}}$ \\
\hline 24 hrs soaking $+24 \mathrm{hr}$ drying & $53^{\circ}$ & $158 \mathrm{E}$ & $190^{\mathrm{AB}}$ & $51^{\mathrm{C}}$ & $30^{\mathrm{GH}}$ & $21^{\mathrm{JK}}$ \\
\hline $\begin{array}{l}12 \mathrm{hrs} \text { soaking }+24 \mathrm{hr} \text { drying }+ \\
12 \mathrm{hrs} \text { resoaking }+24 \mathrm{hr} \text { drying }\end{array}$ & $71^{\mathrm{L}}$ & $158^{\mathrm{E}}$ & $167^{\mathrm{D}}$ & $50^{\mathrm{C}}$ & $28^{\mathrm{HI}}$ & $28^{\mathrm{HI}}$ \\
\hline $\begin{array}{l}18 \mathrm{hrs} \text { soaking }+24 \mathrm{hr} \text { drying }+ \\
18 \mathrm{hrs} \text { resoaking }+24 \mathrm{hr} \text { drying }\end{array}$ & $140^{\mathrm{I}}$ & $160^{\mathrm{DE}}$ & $185^{\mathrm{BC}}$ & $42^{\mathrm{D}}$ & $28^{\mathrm{HI}}$ & $29^{\mathrm{HI}}$ \\
\hline $\begin{array}{l}24 \mathrm{hrs} \text { soaking }+24 \mathrm{hr} \text { drying }+ \\
24 \mathrm{hrs} \text { resoaking }+24 \mathrm{hr} \text { drying }\end{array}$ & $131^{\mathrm{J}}$ & $154^{\mathrm{EFG}}$ & $181^{\mathrm{C}}$ & $24^{\mathrm{IJ}}$ & $37^{\mathrm{DE}}$ & $32^{\mathrm{E}-\mathrm{H}}$ \\
\hline CV (\%) & 12.92 & & & 19.83 & & \\
\hline
\end{tabular}


Table 8. Analysis of variance for the effect of seed treatment and sowing time on plant height, aboveground biomass, thousand seed weight, grain yield, and harvest index of rice at Fogera in the 2010 and 2011 main cropping season.

\begin{tabular}{|c|c|c|c|c|}
\hline \multirow[t]{2}{*}{ Growth and yield parameters } & \multicolumn{4}{|l|}{ Mean square } \\
\hline & Seed treatment (ST) & Sowing date (SD) & ST $x$ SD & Error mean square \\
\hline Plant height & $27.0 \mathrm{NS}$ & $22.6 \mathrm{NS}$ & $19.9 \mathrm{NS}$ & 39.13 \\
\hline Biomass yield & $25.07 *$ & $42.56^{*}$ & $8.25^{*}$ & 0.59 \\
\hline Thousand seeds weight & $2.2 \mathrm{NS}$ & $3.9 \mathrm{NS}$ & $1.1 \mathrm{NS}$ & 1.64 \\
\hline Grain yield & $1.646^{*}$ & $0.193 \mathrm{NS}$ & $0.795^{*}$ & 0.15 \\
\hline Harvest index & $0.017 *$ & $0.013 *$ & $0.008 *$ & 0.003 \\
\hline
\end{tabular}

Table 9. Effect of seed treatment and sowing time on aboveground biomass yield ( $t$ ha-1) and grain yield ( $t$ ha-1) of rice at Fogera in the 2010 and 2011 main cropping season.

\begin{tabular}{|c|c|c|c|c|c|c|}
\hline \multirow{3}{*}{ Seed treatmen } & \multicolumn{3}{|c|}{ Aboveground biomass yield } & \multicolumn{3}{|c|}{ Grain yield } \\
\hline & \multicolumn{3}{|c|}{ Sowing time relative to farmers' time } & \multicolumn{3}{|c|}{ Sowing time relative to farmers' time } \\
\hline & 2 weeks before & 1 week before & $\begin{array}{l}\text { Farmers' sowing } \\
\text { time }\end{array}$ & $\begin{array}{l}2 \text { weeks } \\
\text { before }\end{array}$ & 1 week before & $\begin{array}{l}\text { Farmers' sowing } \\
\text { time }\end{array}$ \\
\hline Dry seed (control) & $9.9^{\mathrm{N}}$ & $10.7^{\mathrm{MN}}$ & $12.0^{\mathrm{F}-\mathrm{J}}$ & $2.35^{\mathrm{J}}$ & $2.37^{\mathrm{J}}$ & $2.56^{\mathrm{HIJ}}$ \\
\hline Pregerminated & $11.3^{\mathrm{J}-\mathrm{M}}$ & $12.8^{\mathrm{B}-\mathrm{F}}$ & $13.7^{\mathrm{A}}$ & $2.3^{\mathrm{J}}$ & $4.08^{\mathrm{BCD}}$ & $4.69^{\mathrm{A}}$ \\
\hline 12 hrs soaking $+24 \mathrm{hr}$ drying & $11.1^{\mathrm{KLM}}$ & $11.2^{\mathrm{J}-\mathrm{M}}$ & $13.2^{\mathrm{A}-\mathrm{D}}$ & $2.41^{\mathrm{J}}$ & $3.58^{\mathrm{FG}}$ & $4.22^{\mathrm{BC}}$ \\
\hline 18 hrs soaking +24 hr drying & $11.6^{\mathrm{I}-\mathrm{L}}$ & $11.7^{\mathrm{H}-\mathrm{L}}$ & $13.0^{\mathrm{A}-\mathrm{E}}$ & $2.45^{\mathrm{J}}$ & $3.49^{\mathrm{G}}$ & $4.24^{\mathrm{BC}}$ \\
\hline 24 hrs soaking +24 hr drying & $12.2^{\mathrm{E}-\mathrm{I}}$ & $12.5^{\mathrm{C}-\mathrm{H}}$ & $13.4^{\mathrm{AB}}$ & $2.57^{\mathrm{HIJ}}$ & $3.72^{\mathrm{D}-\mathrm{G}}$ & $4.44^{\mathrm{AB}}$ \\
\hline $\begin{array}{l}12 \text { hrs soaking }+24 \mathrm{hr} \text { drying }+ \\
12 \text { hrs resoaking }+24 \mathrm{hr} \text { drying }\end{array}$ & $11.8^{\mathrm{G}-\mathrm{L}}$ & $12.4^{\mathrm{D}-\mathrm{I}}$ & $12.6^{\mathrm{B}-\mathrm{G}}$ & $2.54^{\mathrm{HIJ}}$ & $3.76^{\mathrm{D}-\mathrm{G}}$ & $4.05^{\mathrm{B}-\mathrm{E}}$ \\
\hline $\begin{array}{l}18 \text { hrs soaking }+24 \mathrm{hr} \text { drying }+ \\
18 \text { hrs resoaking }+24 \mathrm{hr} \text { drying }\end{array}$ & $12.3^{\mathrm{E}-\mathrm{I}}$ & $12.6^{\mathrm{B}-\mathrm{G}}$ & $12.9^{\mathrm{A}-\mathrm{E}}$ & $2.94^{\mathrm{HI}}$ & $3.98^{\mathrm{C}-\mathrm{F}}$ & $4.00^{\mathrm{B}-\mathrm{F}}$ \\
\hline $\begin{array}{l}24 \mathrm{hrs} \text { soaking }+24 \mathrm{hr} \text { drying }+ \\
24 \mathrm{hrs} \text { resoaking }+24 \mathrm{hr} \text { drying }\end{array}$ & $11.0^{\mathrm{LM}}$ & $11.9^{\mathrm{G}-\mathrm{K}}$ & $13.3^{\mathrm{ABC}}$ & $2.50^{\mathrm{IJ}}$ & $3.62^{\mathrm{EFG}}$ & $4.15^{\mathrm{BCD}}$ \\
\hline CV $(\%)$ & 17.09 & & & 15.63 & & \\
\hline
\end{tabular}

Table 10. Seed treatment and sowing time effects on harvest index of rice at Fogera in the 2010 and 2011 main cropping season.

\begin{tabular}{|c|c|c|c|}
\hline \multirow{2}{*}{ Seed treatment } & \multicolumn{3}{|c|}{ Sowing time relative to farmers' time } \\
\hline & 2 weeks before & 1 week before & Farmers' sowing time \\
\hline Dry seed (control) & $0.24^{\mathrm{B}-\mathrm{F}}$ & $0.22^{\mathrm{DEF}}$ & $0.21^{\mathrm{EF}}$ \\
\hline Pregerminated & $0.2^{\mathrm{F}}$ & $0.32^{\mathrm{ABC}}$ & $0.34^{\mathrm{A}}$ \\
\hline 12 hrs soaking $+24 \mathrm{hr}$ drying & $0.22^{\mathrm{DEF}}$ & $0.32^{\mathrm{ABC}}$ & $0.32^{\mathrm{ABC}}$ \\
\hline 18 hrs soaking +24 hr drying & $0.21^{\mathrm{EF}}$ & $0.3^{\mathrm{A}-\mathrm{E}}$ & $0.33^{\mathrm{AB}}$ \\
\hline 24 hrs soaking +24 hr drying & $0.21^{\mathrm{EF}}$ & $0.3^{\mathrm{A}-\mathrm{E}}$ & $0.33^{\mathrm{AB}}$ \\
\hline $12 \mathrm{hrs}$ soaking $+24 \mathrm{hr}$ drying $+12 \mathrm{hrs}$ resoaking $+24 \mathrm{hr}$ drying & $0.22^{\mathrm{DEF}}$ & $0.3^{\mathrm{A}-\mathrm{E}}$ & $0.32^{\mathrm{ABC}}$ \\
\hline $18 \mathrm{hrs}$ soaking $+24 \mathrm{hr}$ drying $+18 \mathrm{hrs}$ resoaking $+24 \mathrm{hr}$ drying & $0.24^{\mathrm{B}-\mathrm{F}}$ & $0.32^{\mathrm{ABC}}$ & $0.31^{\mathrm{A}-\mathrm{D}}$ \\
\hline 24 hrs soaking $+24 \mathrm{hr}$ drying $+24 \mathrm{hrs}$ resoaking $+24 \mathrm{hr}$ drying & $0.23^{\mathrm{C}-\mathrm{F}}$ & $0.30^{\mathrm{A}-\mathrm{E}}$ & $0.31^{\mathrm{A}-\mathrm{D}}$ \\
\hline CV $(\%)$ & 15.37 & & \\
\hline
\end{tabular}




\section{Discussion}

Sowing pregerminated and primed rice seeds was found to be advantageous in shortening the numbers of emergence, heading, and maturity days. Reduction in the maturity period of rice by 14.1 days is a significant phonological achievement. This achievement could immensely benefit farmers through pre-empting the detrimental effects of terminal moisture stress, which has become a serious rice production bottleneck at Fogera plain due to climate change that caused the occurrence of erratic and scarce rainfall especially at the last phase of the growth period of the plant. Concurrent with the results of this study, Thakur et al. [6] reported faster seed germination and emergence as well as shortened flowering and maturity periods of rice with seed pre-germination. Harris et al. [9] and Farooq et al. [8] also reported similar effects with rice hydro-priming. Ceesay [3] reported that as an escape mechanism to the most critical terminal drought, seed priming along with the use of early maturing varieties were introduced into the rice farming systems of the Gambia. Harris et al. [9] reported that rice seed hydro-priming had significance in that it enables earlier planting of rabi (second) crops as the preceding crop would leave the field earlier.

Under the current practices of dry seed sowing, the rice crop in Fogera plain is having less vegetative growth and LAI. With planting pre-germinated and hydro-primed rice seeds, higher LAI was observed in this experiment. Increased LAI with subsequent increase in rice grain yield was reported earlier by Thakur et al. [6]. Similar to the current results on CGR, Rehman et al. [18] reported an increase in CGR with planting hydro-primed rice seeds. Higher numbers of unproductive tillers and more number of unfilled grains usually associated with terminal moisture stress are common features of the rain-fed lowland rice production of Fogera plain. It was observed from the experiment that the number of unproductive tillers and unfilled spikeletes get much reduced with pre-germination and seed hydro-priming. The results on productive and unproductive tillers and filled and unfilled spikelets obtained in this experiment are in agreement with those of several other researchers $[6,8,9,19,20]$. Several earlier researchers reported that hydro-priming and pre-germinating rice seeds resulted in higher numbers of panicle bearing tillers, higher kernels per panicle, and reduced sterile spikelets compared to dry seed sowing $[6,8,9,19,20]$. Thakur et al. [6] also reported that increase in the physiological activities and yield components associated with seed pre-germination and hydropriming are essential for better survival ability of the crop in the face of terminal moisture stresses.

The higher numbers of unproductive tillers and unfilled spikelets under the farmers' production system were ultimately expressed in the low rice productivity using farmers' practices in the study area. These yield constraining growth parameters were avoided with the practices of seed pre- germination and hydro-priming, both resulting in higher rice grain yield. The increased grain yield and harvest index with planting pre-germinated and hydro-primed seeds in the present study is in line with the results of Kant et al. [20] who reported increased grain yield and harvest index with planting pregerminated rice seeds. In agreement with the results of this study, Harris et al. [9], Farooq et al [8], Rehman et al. [18], Yaril et al. [19] also reported increased rice grain yields and harvest index in response to planting hydro-primed seeds. In this study, yield advantages of $58 \%$ with planting pre-germinated seeds and $50 \%$ with planting hydro-primed seeds were recorded. In agreement with these results, Farooq et al. [8] reported 11-24\% yield advantage over non-primed seeds by planting rice seeds soaked in water for $24 \mathrm{hrs}$ and then air-drying them. They attributed the increase in yield to the increased number of productive tillers and 1000-kernel weight. According to Harris et al. [9], very early capture of resources appears to be the driving factor to some of the benefits associated with seed priming.

In the current study, the observed low yield and growth parameters in response to planting pre-germinated seeds two weeks earlier than the farmers' sowing time indicates the very sensitive nature of pre-germinated seeds to soil moisture stress. The result clearly showed that planting pregerminated seeds obviously need to be carefully synchronized with sufficient soil moisture at planting. In the current study, planting dry seeds at farmers' sowing time resulted in the production of grain yield of $2.56 \mathrm{t}$ ha-1, which is far lower than the best average rice productivity in the area (3.5-4 tha-1). This could be attributed to the terminal moisture stress that particularly occurred in the 2011 cropping season (Table 1). It was clearly observed that seed pregermination and hydro-priming led the crop to finish its phenological growth stages earlier, leading to increased rice yield as the crop escaped the negative effects of terminal moisture stress.

\section{Conclusion}

Terminal moisture stress has been seriously affecting rain-fed lowland rice production in many parts of the world in general and Fogera plain in Ethiopia in particular. Agronomic management practices like seed pre-germination and hydro-priming are believed to be helpful in mitigating terminal droughts. The results of this study revealed that, establishing the rice crop from pre-germinated seeds and planting the seeds at the time of sowing done by farmers led to the production of the highest rice grain yield. In addition, planting hydro-primed rice seed for 24 hours seed soaking and re-drying it for 24 hours at the farmers planting time resulted in the highest grain yield of the crop. Therefore, these two treatments are equally useful in enhancing the grain yield of the crop in the study area. If farmers could carefully follow the pre-germination process and 
synchronize sowing to a time when soil moisture is available in sufficient amounts, seed pre-germination would enable to curb the negative effects of terminal moisture stresses and result in optimum grain yields of the rice crop. The results of this study further imply that, if farmers are unable to carrying out seed pre-germination, seed hydro-priming is the best alternative they could use for enhancing the yield of the crop in the study area. It could, thus, be concluded that, given the erratic nature of rainfall in Ethiopia due to the change in the climatic conditions, subjecting rice seeds to hydro-priming or pre-germination treatments before sowing may contribute substantially to the efforts of increasing food production and household food security in the country.

\section{References}

[1] B.A. Linquist, V. Phengsouvanna and P. Sengxue. Benefits of organic residues and chemical fertilizer to productivity of rain-fed lowland rice and to soil nutrient balances. Nutrient Cycling in Agroecosystems 79:59-72. 2007.

[2] S.M Haefele and B.A.M. Bouman. Drought-prone rainfed lowland rice in Asia: Limitations and management options. In: Serraj J, Bennett J, Hardy B, editors. Drought frontiers in rice: crop improvement for increased rainfed production. Singapore: World Scientific Publishing and Los Baños (Philippines): International Rice Research Institute. Pp 211232. 2009.

[3] M. Ceesay. Management of Rice Production Systems to Increase Productivity in The Gambia, West Africa. A PhD Dissertation, Cornell University, USA. 2004.159 pp.

[4] R.A. Hassanein, A.F. Abdelkader, H. Ali, A.S. Amin and E.M. Rashad. Grain-priming and foliar pretreatment enhanced stress defense in wheat (Triticum aestivum var. Gimaza 9) plants cultivated in drought land. Australian Journal of Crop Science 6(1):121-129. 2012.

[5] A.M. Musa, D. Harris, C. Johansen and J. Kumar. Short Duration Chickpea to Replace Fallow After Aman Rice: The role of on-farm seed priming in the high Barind Tract of Bangladesh. Experimental Agriculture 37: 509-521. 2001.

[6] A.K. Thakur, B.K. James, R. Singh, D.K. Kundu and S. Roychowdhury. Wet seeding in spot: A promising water saving technique for rice cultivation. Research Bulletin No-25, Water Technology Center for Eastern Region (ICAR), Bhubaneswar, India. 2005. 19 pp.

[7] T. Tilahun, Alemayehu A., Minale L. and Zelalem T. Effect of seed soaking and incubation on the emergence, growth and yield of rice. In: Ermias A., Akalu T., Alemayehu A.G., Melaku W., Tilahun T., and Wondimu B.(eds). 2008. Procceedings of the 2nd Annual Regional Conference on Completed Crop Research Activities, 18-21 Sep. 2007. Amhara Regional Agricultural Research Institute. B.D., Ethiopia. 2008. Pp. 36-40.
[8] M. Farooq, K.H.M. Siddique, H. Rehman, T. Aziz, D.J. Lee and A. Wahid. Rice direct seeding: Experiences, challenges and opportunities. Soil and Tillage Research 111:87-98. 2011.

[9] D. Harris, R.S. Tripathi and A. Joshi. On-farm seed priming to improve crop establishment and yield in dry direct-seeded rice. In:Pandey S, Mortimer M, Wade L, Tuong TP, Lopez K, Hardy B, editors. 2002. Direct seeding: research issues and opportunities. Proceedings of the International Workshop on Direct Seeding in Asian Rice Systems: Strategic Research Issues and Opportunities, 25-28 January 2000, Bangkok, Thailand. Los Baños (Philippines): International Rice Research Institute. 2002. Pp 231-239.

[10] M. Farooq, S.M.A. Basra and N. Ahmad. Rice seed priming. International Rice Research Notes 30(2):45-47. 2005.

[11] P.K. Bernard. Evaluation and interpretation of soil analytical data. National Soil service project, FAO/UNDP/ETH/87/010 Ministry of Agriculture, Addis Ababa. 1993. 78Pp.

[12] R.N. Roy, A. Finck, G.J. Blair and H.L.S. Tandon. Plant nutrition for food security. A guide for integrated nutrient management. Food and Agricultural Organization of United Nations. FAO Fertilizer and Plant Nutrition Bulletin 16. Rome. 2006. 348p.

[13] Yoshida S. 1981. Fundamentals of rice crop science The International Rice Research Institute, Los Baños, Laguna, Philippines. 269 pp.

[14] R. Hunt. Plant Growth Analysis. The Inst. of Bio. Studies in Bio. Edward Arnold (Pub.) Ltd. 1978. Pp 8-38.

[15] A. Ahmad, S. Iqbal, S. Ahmad, T. Khaliq, W. Nasim, Z. Husnain, A. Hussain, M. Zia-Ul-Haq and G. Hoogenboom. Seasonal growth, radiation interception, its conversion efficiency and biomass production of Oryza sativa L. Under diverse agroenvironments in Pakistan. Pakistan Journal of Botany 41(3): 1241-1257. 2009.

[16] SAS Institute. SAS Version 9. 1.2 (c) 2002-2003. SAS Institute, Inc., Cary, NC. 2003.

[17] K.A. Gomez and A.A. Gomez. Statistical Procedures for Agricultural Research. 2nd Edn., John Wiley and Sons Inc., New York. 1984. pp 328-332.

[18] H. Rehman, S.M.A. Basra, M. Farooq, N. Ahmed and I. Afzal, Seed priming with $\mathrm{CaCl} 2$ improves the stand establishment, yield and some quality attributes in direct seeded rice (Oryza sativa). International Journal of Agriculture and Biology 13: 786-790. 2011.

[19] L. Yari1, A. Abbasian1, B. Oskouei1 and H. Sadeghi. Effect of seed priming on dry matter, seed size and morphological characters in wheat cultivar. Agriculture and Biology Journal of North America 2(2): 232-238. 2011.

[20] S. Kant, P. Verma and S.S. Pahuja. Growth and yield maintenance in bread wheat by seed priming under late-sown conditions. Acta Agronomica Hungarica 514: 445-453. 2003. 